\title{
Nonlinear effects of energy band structures on optical transitions in quantum dots
}

\author{
Chhi-Chong $\mathrm{Wu}^{\mathrm{a}, *}$, Chau-Jy Lin ${ }^{\mathrm{b}}$ \\ a Institute of Electronics, National Chiao Tung University, Hsinchu, Taiwan \\ ${ }^{\mathrm{b}}$ Department of Applied Mathematics, National Chiao Tung University, Hsinchu, Taiwan
}

\begin{abstract}
The quantum theory of nonlinear effects for optical transitions of electrons in quasi-zero dimensional (Q0D) quantum dots fabricated from n-type III-V compound semiconductor materials such as n-type GaAs has been studied due to the nonparabolicity of energy band structures. We use the effective mass approximation for carriers in the quantum dots. Most realistic quantum dot systems contain the box with a thickness $c$ and the lateral width $(a, b)$. Using the time-independent perturbation theory, the first order correction of the eigenfunctions and eigenvalues for the system has been calculated. And the free-carrier absorption coefficient may be calculated for Q0D quantum dots from n-type GaAs where the polar optical phonon scattering is dominant. Our results show that the free-carrier absorption coefficient in Q0D quantum dots increases quite rapidly with increasing temperature in the region of low temperatures. When temperatures are larger than $100 \mathrm{~K}$, the free-carrier absorption coefficient increases slowly with temperature. This shows that the nonlinear property of energy band structures due to the nonparabolicity plays an important role in low temperatures. The discussion about the dot size effect of the quantum confinement region in n-type GaAs quantum dots has also been given. (C) 2002 Published by Elsevier Science B.V.
\end{abstract}

Keywords: Optical transition; Quantum dot system; Polar optical phonon scattering

\section{Introduction}

Low-dimensional quantum nanostructures such as quantum wires and quantum dots have attracted considerable attention in view of their basic physics and potential device applications [13]. Especially, the interesting things in the fabrication of low-dimensional semiconductor structures are the modified electronic and optical properties of these structures, which are controllable to a certain degree through the flexibility in the structure design. These features make quantum

\footnotetext{
*Corresponding author. Fax: + 886-35-724361.

E-mail address: chhiwu@cc.nctu.edu.tw (C.-C. Wu).
}

confined semiconductors very promising for possible device applications in microelectronics, nonlinear optics, and many other fields. The electron confinement in low-dimensional structures modifies the density of states and enhances the Coulomb interaction of electrons. In this work, electrons in a quantum dot are confined by a heterostructure of compound semiconductors such as $\mathrm{Al}_{0.45} \mathrm{Ga}_{0.55} \mathrm{As} / \mathrm{GaAs}$.

\section{Theory}

As a quantum dot is assumed to be a well with infinitely high-energy barriers, the effective 
Hamiltonian for the nonparabolic energy band can be expressed as

$$
\begin{aligned}
H_{\mathrm{eff}}= & H\left(1+\frac{H}{E_{\mathrm{g}}}\right)=\sum_{i}\left[\frac{\mathbf{p}_{i}^{2}}{2 m^{*}}+V_{0}\left(\mathbf{r}_{\mathrm{i}}\right)\right] \\
& +\frac{1}{2} \sum_{i \neq j} \frac{e^{2}}{\varepsilon\left|\mathbf{r}_{i}-\mathbf{r}_{j}\right|},
\end{aligned}
$$

where $E_{\mathrm{g}}$ is the energy gap between the conduction and valence bands, and $V_{0}\left(\mathbf{r}_{i}\right)$ is an infinite potential defined as follows:

$$
V_{0}=\left\{\begin{array}{cc}
0 & \text { for }|x|<a,|y|<b,|z|<c, \\
\infty & \text { for }|x|>a,|y|>b,|z|>c .
\end{array}\right.
$$

Using the time-independent perturbation theory, the eigenfunctions and eigenvalues for the system are given by

$$
\begin{aligned}
\Psi_{\ell m n}(\mathbf{r})= & \left(\frac{8}{a b c}\right)^{1 / 2} \sin \left(\frac{\pi \ell x}{a}\right) \sin \left(\frac{\pi m y}{b}\right) \\
& \times \sin \left(\frac{\pi n z}{c}\right)+\frac{2 \sqrt{2} \mathrm{e}^{2}}{\pi^{2}(a b c)^{3 / 2} \varepsilon E_{\mathrm{g}}} \\
& \times \sum_{\substack{\left(\ell^{\prime}, m^{\prime}, n^{\prime}\right) \\
\neq(\ell, m, n)}} P\left(\ell^{\prime}-\ell, m^{\prime}-m, n^{\prime}-n\right) \\
& \times Q\left(\ell, m, n ; \ell^{\prime}, m^{\prime}, n^{\prime}\right) \sin \left(\frac{\pi \ell^{\prime} x}{a}\right) \\
& \times \sin \left(\frac{\pi m^{\prime} y}{b}\right) \sin \left(\frac{\pi n^{\prime} z}{c}\right)
\end{aligned}
$$

and

$E_{\ell m n}=-\frac{1}{2}\left\{1-\left[1+\frac{2 \pi^{2} \hbar^{2}}{m^{*} E_{\mathrm{g}}}\left(\frac{\ell^{2}}{a^{2}}+\frac{m^{2}}{b^{2}}+\frac{n^{2}}{c^{2}}\right)\right]^{1 / 2}\right\}$,

where $P(\ell, m, n)$ and $Q\left(\ell, m, n ; \ell^{\prime}, m^{\prime}, n^{\prime}\right)$ are functions of $\ell, m, n$ and $\ell^{\prime}, m^{\prime}, n^{\prime}$ with parameters $a, b$, and $c$.

The electron-polar-optical-phonon interaction potential is given by $[4,5]$

$$
\begin{aligned}
U_{s}= & \operatorname{ie} \omega\left(\frac{4 \pi}{\varepsilon^{\prime} V}\right)^{1 / 2} \sum_{\mathbf{q}} \frac{\mathbf{q} \cdot \hat{\varepsilon}_{\mathbf{q}}}{|\mathbf{q}|^{2}}\left[\exp (-\mathrm{iq} \cdot \mathbf{r}) Q_{\mathbf{q}}^{*}\right. \\
& \left.\times \exp (\mathrm{iq} \cdot \mathbf{r}) Q_{\mathbf{q}}\right],
\end{aligned}
$$

where $\mathbf{q}$ is the wave vector of the polar optical phonons, $\hat{\varepsilon}_{\mathbf{q}}$ is their polarization vector, and $\varepsilon^{\prime}=\left(1 / \varepsilon_{\infty}-1 / \varepsilon\right)^{-1}$. Here $\varepsilon_{\infty}$ and $\varepsilon$ are the high-frequency and static dielectric constants of semiconductors, respectively, $Q_{\mathbf{q}}^{*}$ and $Q_{\mathbf{q}}$ are creation and annihilation operators for the optical phonons. The matrix element of the electron-photon interaction Hamiltonian is

$$
\begin{aligned}
\left\langle\ell^{\prime} m^{\prime} n^{\prime}\left|H_{\mathrm{rad}}\right| \ell m n\right\rangle= & -\frac{\sqrt{2} \pi^{3 / 2} \hbar^{3 / 2}}{m^{*}}\left(\frac{n_{0}}{\varepsilon \Omega a b c}\right)^{1 / 2} \\
& \times\left(\frac{\ell^{2}}{a^{2}}+\frac{m^{2}}{b^{2}}+\frac{n^{2}}{c^{2}}\right)^{1 / 2} \\
& \times \delta_{\ell^{\prime}, \ell} \delta_{m^{\prime}, m} \delta_{n^{\prime}, n}
\end{aligned}
$$

where $n_{0}$ is the number of photons in the radiation field and $\Omega$ is the photon frequency. The electron distribution function in a nondegenerate semiconductor can be expressed as

$f_{\ell m n}=n_{\mathrm{e}} \mathrm{e}^{-E_{\ell m n} / k_{\mathrm{B}} T}\left(\sum_{\ell m n} \mathrm{e}^{-E_{\ell m n} / k_{\mathrm{B}} T}\right)^{-1}$,

where $n_{\mathrm{e}}$ is the concentration of electrons in solids. The free-carrier absorption coefficient can be obtained as

$$
\begin{aligned}
\alpha= & \frac{8 \pi^{3} \mathrm{e}^{4}}{\varepsilon^{1 / 2} \varepsilon^{\prime} m^{*} a b c v_{\mathrm{c}} \Omega}\left[\exp \left(\frac{\hbar \omega}{k_{\mathrm{B}}}\right)-1\right]^{-1} \\
& \times\left[\sum_{\ell, m, n} \exp \left(-\frac{E_{\ell m n}}{k_{\mathrm{B}} T}\right)\right]^{-1} \\
& \times \sum_{\ell_{f}>\ell_{i}=1} \sum_{m_{f}>m_{i}=1} \sum_{n_{f}>n_{i}=1} \mathrm{e}^{-E_{\ell_{i} m_{i} n_{i} / k_{\mathrm{B}} T}} \\
& \times\left\{\left(\frac{\ell_{f}^{2}}{a^{2}}+\frac{m_{f}^{2}}{b^{2}}+\frac{n_{f}^{2}}{c^{2}}\right)\left[(\Omega+2 \omega)^{-2}+\Omega^{-2}\right]\right. \\
& +\left(\frac{\ell_{i}^{2}}{a^{2}}+\frac{m_{i}^{2}}{b^{2}}+\frac{n_{i}^{2}}{c^{2}}\right)\left[(2 \Omega+\omega)^{-2}+(2 \Omega-\omega)^{-2}\right] \\
& +2\left(\frac{\ell_{i}^{2}}{a^{2}}+\frac{m_{i}^{2}}{b^{2}}+\frac{n_{i}^{2}}{c^{2}}\right)\left(\frac{\ell_{f}^{2}}{a^{2}}+\frac{m_{f}^{2}}{b^{2}}+\frac{n_{f}^{2}}{c^{2}}\right)^{1 / 2} \\
& \left.\times\left[\frac{1}{(\Omega+2 \omega)(2 \Omega+\omega)}+\frac{1}{\Omega(2 \Omega-\omega)}\right]\right\}
\end{aligned}
$$




$$
\begin{aligned}
& \times\left\{\left[\frac{\left(\ell_{f}-\ell_{i}\right)^{2}}{a^{2}}+\frac{\left(m_{f}-m_{i}\right)^{2}}{b^{2}}+\frac{\left(n_{f}-n_{i}\right)^{2}}{c^{2}}\right]^{-1}\right. \\
& \left.\times\left[\frac{\left(\ell_{f}+\ell_{i}\right)^{2}}{a^{2}}+\frac{\left(m_{f}+m_{i}\right)^{2}}{b^{2}}+\frac{\left(n_{f}+n_{i}\right)^{2}}{c^{2}}\right]^{-1}\right\},
\end{aligned}
$$

where $v_{\mathrm{c}}$ is the velocity of light.

\section{Numerical results}

As a numerical example, we consider the freecarrier absorption coefficient in n-type GaAs with the optical phonon frequency $\omega=5.5 \times 10^{13} \mathrm{rad} / \mathrm{s}$. The relevant values of physical parameters are taken to be [6] $n_{\mathrm{e}}=1.73 \times 10^{15} \mathrm{~cm}^{-3}, m^{*}=0.07 m_{0}$

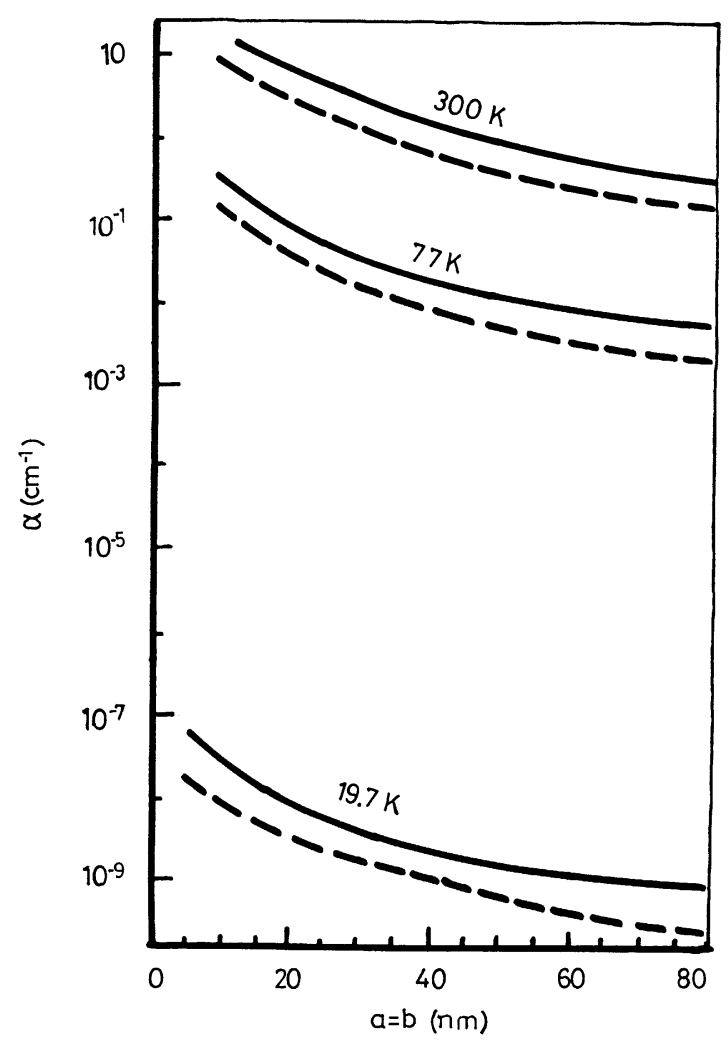

Fig. 1. Free-carrier absorption coefficient as a function of lateral width. Solid curves: $c=10 \mathrm{~nm}$; broken curves: $c=20 \mathrm{~nm}$.
( $m_{0}$ is the mass of free electrons), $\varepsilon=12.9, \varepsilon_{\infty}=$ 11.1 and $E_{\mathrm{g}}=1.51 \mathrm{eV}$. We plot the free-carrier absorption coefficient $\alpha$ as a function of lateral width $a=b$ as shown in Fig. 1 with the photon frequency $\Omega=53.4 \mathrm{THz}$ (or $5.6 \mu \mathrm{m}$ wavelength of a $\mathrm{CO}_{2}$ laser). It is shown that $\alpha$ decreases with increasing the lateral width $a=b$ or the film thickness $c$. It can also be seen that $\alpha$ increases with temperature. This is the same property as that for the acoustic phonon scattering [7] and for the polar optical phonon scattering [6] in quasi-twodimensional structures. In Fig. 2, we plot $\alpha$ as a function of the photon frequency $\Omega$ with the lateral width $a=b=20 \mathrm{~nm}$. It shows that $\alpha$ decreases monotonically with increasing photon frequency. In Fig. 3, $\alpha$ is plotted as a function of temperature with $\Omega=53.4 \mathrm{THz}$ and the film thickness $c=10 \mathrm{~nm}$. It shows that $\alpha$ increases rapidly with

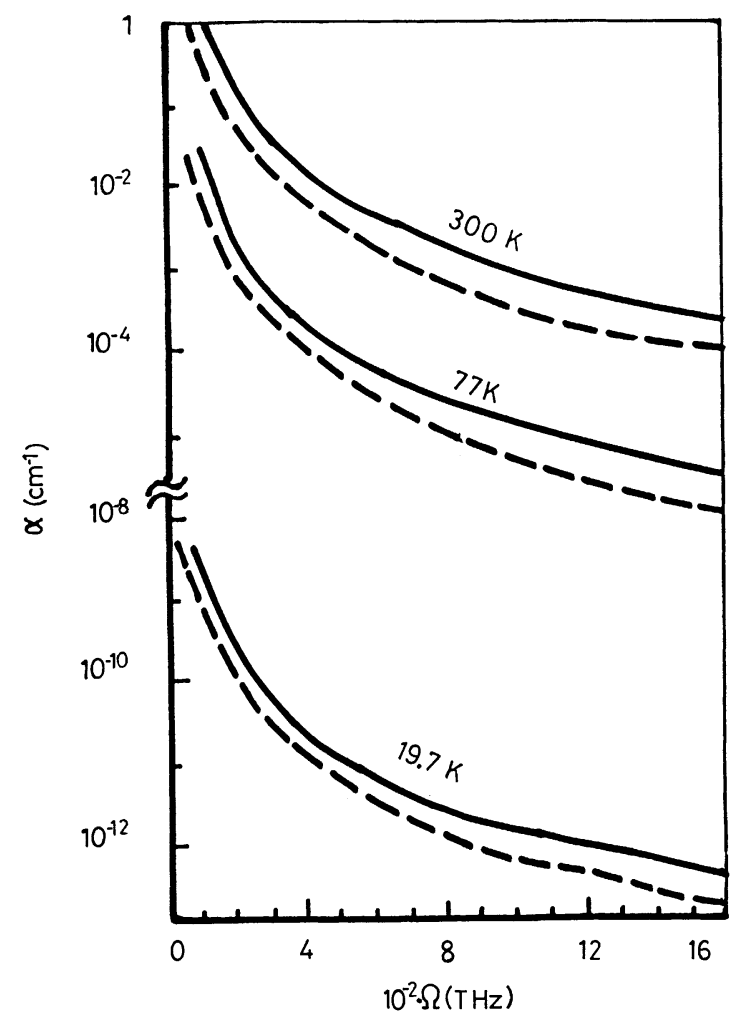

Fig. 2. Free-carrier absorption coefficient as a function of photon frequency. Solid curves: $c=10 \mathrm{~nm}$; broken curves: $c=20 \mathrm{~nm}$. 


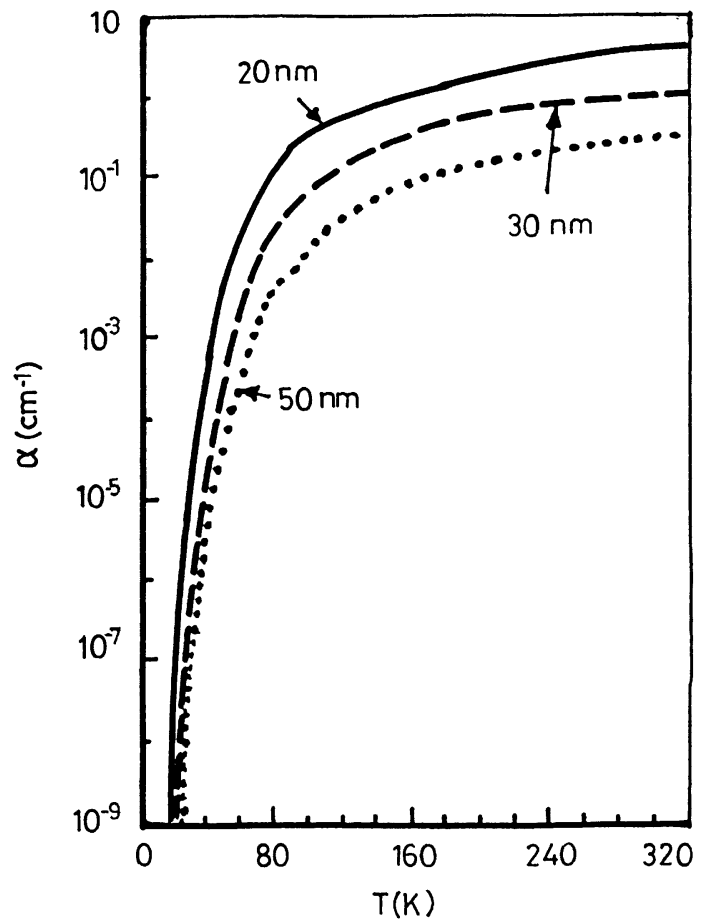

Fig. 3. Free-carrier absorption coefficient as a function of temperature with $\Omega=53.4 \mathrm{THz}$ and $c=10 \mathrm{~nm}$. temperature in the lower-temperature region, and then increases slowly with temperature in the higher-temperature region.

\section{Acknowledgements}

This study was supported by the National Science Council, Republic of China, under contract number NSC 89-2215-E-009-061.

\section{References}

[1] H. Sakaki, Jpn. J. Appl. Phys. 19 (1980) L735.

[2] Y. Arakawa, H. Sakaki, Appl. Phys. Lett. 40 (1982) 939.

[3] H. Gotoh, H. Ando, H. Kanbe, Appl. Phys. Lett. 68 (1996) 2123.

[4] S. Das Sarma, A. Madhukar, Phys. Rev. B 22 (1980) 2823.

[5] P.J. Price, Ann. Phys. 133 (1981) 217.

[6] C.C. Wu, C.J. Lin, J. Appl. Phys. 79 (1996) 781.

[7] C.C. Wu, C.J. Lin, Physica B 219\&220 (1996) 65. 